Nicolas Le Novère Alain Bessis Clément Léna Marina Piccioto Michele Zoli

\section{ADRESSE}

N. Le Novère : étudiant à Paris VI. A. Bessis : boursier de la fondation Singer-Polignac. C. Léna: boursier de l'Education nationale. M. Piccioto: boursière Human Frontiers. M. Zoli : sous-directeur au Collège de France. Laboratoire de ncurobiologie moléculaire, bâtiment des Biotechnologies, Institut Pas teur, 25, rue du Dr-Roux, 75015 Paris, France.

\title{
Le récepteur nicotinique neuronal de l'acétylcholine : du gène au tabagisme
}

Le récepteur nicotinique musculaire de l'acétylcholine est une protéine allostérique pentamérique, prototype de la superfamille des canaux ioniques activés par des neuromédiateurs. Une famille de récepteurs apparentés est localisée dans le système nerveux. Ces récepteurs, composés de deux sous-unités $\alpha$ engagées dans la liaison de l'acétylcholine, et de trois sous-unités non $\alpha$, sont les agents moléculaires de la transmission nicotinique neuronale. Une dizaine de gènes codant pour des sous-unités sont actuellement identifiés et transcrits différemment selon la localisation anatomique. Les propriétés électrophysiologiques et pharmacologiques du récepteur dépendent de sa composition en sous-unités et sont modifiées par l'innervation ainsi que par diverses substances comme le $\mathrm{Ca}^{2+}$ et l'AMP cyclique. L'expression du récepteur nicotinique neuronal de l'acétylcholine est altérée dans diverses maladies.

L 'acétylcholine (ACh) sert de neuromédiateur dans la transmission synaptique au niveau du système nerveux, central et périphérique. On distingue deux types de récepteur cholinergique. Le premier, dit muscarinique, fait partie de la famille des récepteurs métabotropiques à sept hélices transmembranaires, liés aux protéines G. Le second, qui est activé par la nicotine et qui fait l'objet de cet article, appartient à la superfamille des canaux ioniques dont l'ouverture est activée par des neuromédiateurs [1]. Ce groupe de protéines comprend les récepteurs nicotiniques de l'acétylcholine, le récepteur de la glycine, le récepteur du GABA de type $A$, les récepteurs du glutamate de type NMDA et AMPA ainsi que celui de la sérotonine 5HT3, qui possèdent certains motifs moléculaires et physiologiques communs, notamment quatre segments hydrophobes transmembranaires (revue dans [2]). Les homologies de séquence entre les différents membres de la superfamille permettent de penser qu'ils dérivent d'un ancêtre commun [3].

\section{Structure du récepteur nicotinique musculaire}

Le récepteur nicotinique de l'acétylcholine (nAChR) est présent dans l'organe électrique de gymnote ou de poisson torpille en quantité importante, ce qui a permis sa purification et son étude par des méthodes physico-chimiques. Un récepteur très voisin est responsable de la transmission de l'influx nerveux à la jonction neuromusculaire. Ces circonstances 


\section{RÉFÉRENCES}

1. Changeux JP. Functional architecture and dynamics of the nicotinic acetylcholine receptor: an allosteric ligand-gated ion channel. Fidia Res Found Neurosci Award Lectures $1990 ; 4: 21-168$.

2. Betz H. Ligand-gated ion channels in the brain : the amino-acid receptor superfamily. Neuron 1990 ; 5 : 383-92.

3. Cockroft VB, Osgutjorpe DJ, Barnard EA, Friday AE, Lunt GG. Ligandgated ion channels : homology and diversity. Mol Neurobiol 1992 ; 4 : 129-69.

4. Giraudat J, Dennis $M$, Heidmann $T$, Chang JY, Changeux JP. Structure of the high-affinity binding site for noncompetitive blockers of the acetylcholine receptor : serine-262 of the subunit is labeled by $\left[{ }^{3} \mathrm{H}\right]$ chlorpromazine. Proc Natl Acad Sci USA 1986 ; 83 : 2719-23.

5. Hucho F, Oberthür W, Lottspeich F. The ion channel of the nicotinic acetylcholine receptor is formed by the homologous helices MII of the receptor subunits. FEBS Lett 1986 ; 205 : 137-42.

6. Imoto $\mathrm{K}$, Methfessel C, Sakmann B, Mishima M, Mori Y, Komo T, Fukuda K, Kurasaki M, Bujo H, Fujita Y, Numa S. Location of a $\delta$-subunit region determining ion transport through the acetylcholine receptor channel. Nature 1986 ; 324: 670-4.

7. Dennis M, Giraudat J, KotzybaHibert F, Goeldner M, Hirth C, Chang JY, Lazure C, Chrétien M, Changeux JP. Amino-acids of the Torpedo marmorata acetylcholine receptor $\alpha$ subunit labeled by a photoaffinity ligand for the acetylcholine binding site. Biochemistry $1988 ; 27$ : 2346-57.

8. Patrick J, Stallcup WB. Immunological distinction between acetylcholine receptor and the $\alpha$-bungarotoxine binding component on sympathetic neurons. Proc Natl Acad Sci USA 1977 ; 74 : 4689-92.

9. Clarke PBS, Schwartz RD, Paul SM, Pert CB, Pert A. Nicotinic binding in rat brain : autoradiographic comparison of $\left[{ }^{3} \mathrm{H}\right]$ acetylcholine, $\left[{ }^{3} \mathrm{H}\right]$ nicotine, and [ $\left.{ }^{125} \mathrm{I}\right] \alpha$-bungarotoxin. J Neurosci $1985 ; 5$ : 1307-15.

10. Couturier S, Bertrand D, Matter JM, Hernandez MC, Bertrand S, Millar N, Valera S, Barkas T, Ballivet M. A neuronal nicotinic acetylcholine receptor subunit $(\alpha 7)$ is developmentally regulated and forms a homo-oligomeric channel blocked by $\alpha$ BTX. Neuron $1990 ; 5$ : 847-56.

11. Boulter J, O'Shea-Greenfield A, Duvoisin RM, Connolly JG, Wada E, Jensen A, Gardner PD, Ballivet M, Denens ES, Mc Kinnon D, Heinemann S, Patrick J. $\alpha 3, \alpha 5$ and $\beta 4$ : three members of the rat neuronal nicotinic acetylcholine receptor-related gene family form a gene cluster. J Biol Chem 1990 : $265: 4472-82$. ont fait du nAChR de l'organe électrique et du muscle le prototype de la superfamille des canaux ioniques réglés par un neuromédiateur (pour une revue exhaustive, voir [1]).

Le nAChR musculaire est une protéine allostérique pentamérique de 300000 daltons formée de deux sousunités $\alpha 1$ portant le site de fixation de l'acétylcholine (figure $1 A$ ) et de trois sous-unités non $\alpha$ nommées $\beta 1$, $\gamma$ et $\delta$. Il contient un canal cationique non spécifique dont l'ouverture provoque, dans les conditions physiologiques, un courant entrant rapide. Le profil hydropathique de chaque sous-unité révèle une longue séquence hydrophile amino-terminale, trois segments hydrophobes (MI-MIII), suivis d'un domaine hydrophile variable puis d'un quatrième segment hydrophobe (MIV). Selon la disposition la plus couramment admise de chaque chaîne, le grand domaine hydrophile est extracellulaire, glycosylé et ancré dans la membrane par les quatre segments transmembranaires organisés au moins en partie en hélices $\alpha$, la boucle hydrophile située entre les hélices MIII et MIV étant exposée sur la face cytoplasmique de la membrane (figure 1B). Les expériences de marquage par des bloquants non compétitifs photoactivables $[4,5]$ et de mutagenèse dirigée [6] montrent que le canal ionique est délimité par l'assemblage des cinq régions transmembranaires MII de chaque sousunité (figure 2A). Dans toutes les sousunités, le grand domaine hydrophile amino-terminal contient deux cystéines espacées de treize résidus définissant la boucle glycosylée, indispensable au maintien de la structure spatiale correcte. Des expériences de marquage d'affinité développées avec des antagonistes et agonistes photoactivables compétitifs de l'ACh permettent de marquer les tyrosines 93, 151, 190 et 198, les tryptophanes 86 et 149 ainsi que le doublet de cystéines 192 et 193 de la sous-unité $\alpha 1$ [7]. Ces résultats suggèrent que le site de liaison de l'ACh est formé à partir de trois boucles du grand domaine amino-terminal (figure $2 B$ ).

Dès 1977, des sites nicotiniques distincts du récepteur musculaire étaient mis en évidence dans le système nerveux périphérique [8] puis, ultérieurement, dans le système nerveux cen- tral, où existe une grande diversité de récepteurs nicotiniques neuronaux [9].

\section{Les sous-unités du récepteur nicotinique de l'acétylcholine définissent une famille génique}

Le premier $\mathrm{ADNc}$ correspondant à une sous-unité nicotinique centrale a été isolé par hybridation croisée avec une sonde musculaire. Des méthodes similaires ont conduit à l'identification d'une dizaine de gènes (revue dans [10]), présents dans des groupes animaux aussi différents que les insectes et les mammifères. La localisation chromosomique de certains de ces gènes a été effectuée et révèle parfois une disposition groupée $[11,12]$. Ils se répartissent en deux sousfamilles, $\alpha(\alpha 2$ à $\alpha 7)$ ou non $\alpha(\beta 2$ à $\beta 4$ ), selon qu'ils codent ou non pour le doublet de cystéines dans le domaine amino-terminal. Les séquences primaires des sous-unités neuronales sont très proches de celles des sous-unités musculaires et tous les motifs fonctionnellement importants sont conservés (figure 1). Sur la sousunité $\alpha 7$, les mutations des acides aminés homologues aux résidus Tyr93, Trp149 et Tyr190 de la sousunité $\alpha$ musculaire modifient l'affinité pour l'ACh du récepteur exprimé dans l'oocyte [13]. Ce résultat montre que le site de fixation de l'ACh du récepteur neuronal est situé, comme pour le récepteur musculaire, en partie sur les sous-unités $\alpha$ (figure 1A).

La stœchiométrie des sous-unités du récepteur a été déterminée par deux approches différentes. D'une part, l'expression, dans les oocytes [14] et dans les fibroblastes, de récepteurs marqués au soufre 35 met en évidence un rapport $\beta / \alpha$ peu différent de 1,5 . D'autre part, la mutagenèse dirigée permet d'obtenir des sous-unités conférant des conductances différentes au récepteur qu'elles forment. $\mathrm{La}$ co-injection de sous-unités mutantes et sauvages conduit à l'apparition de récepteurs avec des conductances sauvages, hybrides et mutées. Du nombre de conductances hybrides on déduit que la stœchiométrie est $\alpha \mathrm{i}_{2} \beta \mathrm{j}_{3}$ [15]. 


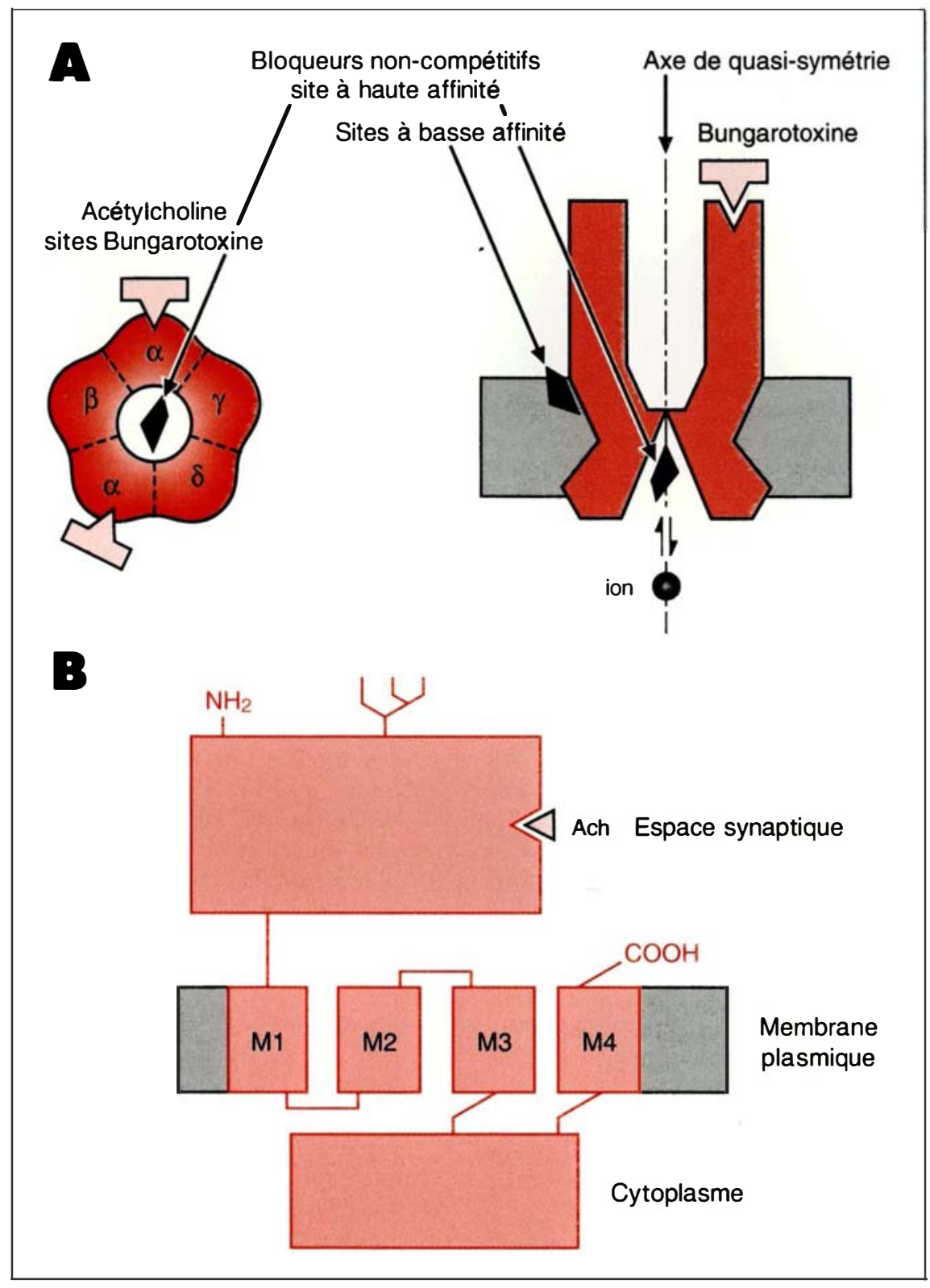

Figure 1. A. Structure du récepteur nicotinique, vue en coupe et de dessus. B. Organisation d'une sous-unité $\alpha$ de récepteur nicotinique.

Parallèlement, la purification du récepteur provenant du cerveau de poulet à l'aide d'un anticorps monoclonal spécifique de l'organe électrique de torpille (MAb35) a montré que les sous-unités $\alpha 2,3,4$ et $\beta 2$ étaient exprimées in vivo. Un anticorps monoclonal dirigé contre $\beta 2$ de poulet (MAb270) précipite $90 \%$ des sites nicotiniques purifiés, ce qui prouve que $\beta 2$ est une sous-unité présente dans la majorité des récepteurs. Utilisé sur le cerveau de rat, l'anticorps MAb270 permet d'isoler un récepteur formé de sous-unités de 52 $\mathrm{m} / \mathrm{s} n^{\circ} 1$ vol. 9, janvier 93 encore bien caractérisés, mais leurs propriétés pharmacologiques commencent à être connues [19], révélant des comportements originaux et diversifiés.

\section{La diversité structurale entraîne une hétérogénéité fonctionnelle}

Agonistes et antagonistes permettent de distinguer plusieurs types de récepteurs nicotiniques (Tableau I). Le récepteur musculaire est plus efficacement activé par la suberyldicholine que par l'ACh et inversement pour le récepteur neuronal. De même, le décaméthonium bloque mieux la réponse nicotinique dans le muscle que dans les ganglions autonomes [20]. Des différences existent également entre différentes structures nerveuses : par exemple, l'ordre d'efficacité des agonistes dans l'habénula est nicotine, cytisine puis $\mathrm{ACh}$ alors que dans le noyau interpédonculaire, on trouve cytisine, $\mathrm{ACh}$ puis nicotine. Il est maintenant clair que la composition du récepteur en sousunités est responsable de ces différences. Un récepteur $\alpha \mathrm{i} \beta 4$ est sensible à la cytisine alors que les récepteurs $\alpha \mathrm{i} \beta 2$ sont insensibles à cette molécule [21].

Les toxines de venin de serpent sont des bloquants compétitifs du nAChR qui ont joué un rôle de premier ordre dans les études du récepteur musculaire. L' $\alpha$-bungarotoxine $(\alpha$-Bgt), provenant du serpent Bungarus multicinctus et qui se lie de manière quasi irréversible au récepteur musculaire, bloque les réponses nicotiniques dans des cellules de l'hippocampe en culture [22] et dans le cervelet [23]. Un anticorps monoclonal dirigé contre les récepteurs liant l' $\alpha$-Bgt dans le cerveau précipite la sous-unité $\alpha 7$, mais ni $\alpha 4$ ni $\beta 2$, et, inversement, un anticorps contre $\alpha 4$ ou $\beta 2$ ne précipite pas la sous-unité $\alpha 7$. $\alpha 4$ et $\beta 2$, au contraire d' $\alpha 7$, ne font donc pas par-

- Un travail récent montre cependant que la sousunité $\alpha 5$ est précipitée avec des récepteurs nicotiniques dans le cerveau. Toutefois, la présence d'une autre sous-unité $\alpha$ (en l'occurrence $\alpha 3$ ou $\alpha 4$ ) serait indispensable, ce qui met en évidence une nouvelle classe de récepleurs neuronaux, formés d'au moins trois sousunités différentes [54]. 


\section{RÉFÉRENCES}

12. Bessis A, Simon-Chazottes D, DevillersThiéry A, Guénct JL, Changcux JP. Chromosomal localization of the mouse genes coding for $\alpha 2, \alpha 3, \alpha 4$ and $\beta 2$ subunits of ncuronal nicotinic acetylcholine receptor. FEBS Lett 1990 ; 264 : 48-52.

13. Galzi JL, Bertrand D, DevillersThiéry A, Revah F, Bertrand S, Changcux JP. Functional significance of aromatic amino acids from the threc peptide loops of the $\alpha 7$ ncuronal nicotinic receptor site investigated by site-directed mutagenesis. FEBS Lett 1991 ; 294 : 198-202.

14. Anand R, Conroy WG, Schocpfer R, Whiting $P$, Lindstrom J. Neuronal nicotinic acctylcholine receptors expressed in Xenopus oocytes have a pentameric quaternary structurc. J Biol Chem 1991; 266 : 11192-8.

15. Cooper E, Couturier S, Ballivet $M$. Pentameric structure and subunit stoichiometry of a ncuronal acetylcholine receptor. Nature $1991 ; 350$ : 235-8.

16. Whitings $P$, Lindstrom J. Purification and characterization of a nicotinic acctylcholine reccptor from rat brain. Proc Natl Acad Sci USA 1987 ; 84 : 595-9.

17. Dencris ES, Boulter J, Swanson LW, Patrick J, Heinemann S. $\beta 3$ : a new member of nicotinic acetylcholine receptor gene family is expressed in brain. J Biol Chem $1989 ; 264: 6268-72$.

18. Revah F, Bertrand D, Galzi JL, Devillers-Thiéry A, Mulle C, Hussy N, Bertrand S, Ballivet M, Changeux JP. Mutations in the channel domain alter desensitization of a ncuronal nicotinic receptor. Nature 1991 ; 353 : 846-9.

19. Mulle C, Vidal C, Benoit P, Changeux JP. Existence of different subtypes of nicotinic acetylcholine receptors in the habenulo-interpeduncular system. J Neurosci $1991 ; 11: 2588-97$

20. Paton WDM, Zaimis EJ. The pharmacological actions of polymethylene bistrimethylammonium salts. BrJ Pharmacol $1949 ; 4$ : 381-400.

21. Luctje CW, Patrick J. Both $\alpha$ - and $\beta$ subunits contribute to the agonist sensitivity of the ncuronal nicotinic acetylcholine receptor. J Neurosci 1991; 11 : 837-45.

22. Alkondon $\mathbf{M}$, Albuquerque EX. Initial charactcrization of the nicotinic acetylcholine receptors in the rat hippocampal ncurons. J Recept Res 1991; 11: 1001-21.

23. De la Garza R, McGuirc TJ, Frecdman R, Hoffer BJ. Sclective antagonism of nicotine actions in the rat cercbellum with $\alpha$-bungarotoxin. Neuroscience $1987 ; 23$ :

\begin{tabular}{|c|c|c|c|c|}
\hline & $\begin{array}{l}\text { PROP } \\
\text { DE DIF }\end{array}$ & $\begin{array}{l}\text { ableac } \\
\text { HARN } \\
\text { nAC }\end{array}$ & $\begin{array}{l}\text { JGIQUES } \\
\text { JRONAUX }\end{array}$ & \\
\hline $\begin{array}{l}\text { Composition } \\
\text { donnée } \\
\text { du récepteur }\end{array}$ & $\begin{array}{r}\text { Concen } \\
\text { ACh }\end{array}$ & $\begin{array}{r}\text { lative } \\
\text { Nic }\end{array}$ & $\begin{array}{l}\text { iste nécessa } \\
\text { Cyt }\end{array}$ & $\begin{array}{l}\text { un effet } \\
\text { DMPP }\end{array}$ \\
\hline$\alpha 2 \beta 2$ & 1 & 0,2 & 14 & 1 \\
\hline$\alpha 2 \beta 4$ & 1 & 0,4 & 0,07 & 5 \\
\hline$\alpha 3 \beta 2$ & 1 & 17 & $>100$ & 1 \\
\hline$\alpha 3 \beta 4$ & 1 & 1 & 0,4 & 1 \\
\hline$\alpha 4 \beta 2$ & 1 & 1 & $>30$ & 4 \\
\hline$\alpha 4 \beta 4$ & 1 & 0,3 & 0,06 & 24 \\
\hline
\end{tabular}

Contribution des sous-unités $\alpha$ et $\beta$ à la sensibilité aux agonistes. ACh : acétylcholine ; Nic: nicotine; Cyt : cytisine; DMPP : 1,1-diméthyl-4-phénylpipérazinium. (Adapté de (211.)

tie des sous-unités liant l' $\alpha$-Bgt [24]. La n-bungarotoxine (appeléc encore $\kappa$-bungarotoxine, bungarotoxine 3.1 ou toxine F) agit sur les ganglions périphériques [25] et bloque certaines réponses nicotiniques centrales [26]. Comme pour les agonistes compétitifs, la composition en sous-unités des réceptcurs détermine l'efficacité de la toxine : $\alpha 3$ et $\beta 2$ entraînent une plus grande sensibilité que $\alpha 2, \alpha 4$ et $\beta 2$ [27].

La distinction initiale entre la sousunité $\alpha$, responsable de la liaison de l'effecteur, et $\beta$, qui n'aurait qu'un rôle structural, ne peut être maintenue, cette dernière influençant la fixation des ligands compétitifs. Cette constatation est à rapprocher de la conservation de certains acides aminés du site de liaison de l'ACh dans les sous-unités $\beta$ (figure $2 B$ ). La sensibilité différentielle aux ligands des récepteurs selon les combinaisons de sous-unités dont ils sont composés laisse entrevoir l'esquisse d'une pharmacologie ciblée, d'autant que les études anatomiques révèlent des répartitions très variées selon les sousunités.

\section{La répartition anatomique varie d'une sous-unité} à l'autre

La localisation des sites nicotiniques à l'aide de ligands radioactifs dans le ccrveau montre une relative ségrégation entre les sites de la nicotine et de l'ACh (après blocage des sites muscariniques) et ceux liant l' $\alpha$-Bgt [9]. Par exemple, le thalamus n'est marqué que par l'ACh et la nicotine tandis que l'hippocampe ne présente un marquage fort qu'avec l' $\alpha$-Bgt. Quand on mesure la consommation de glucose lors de la perfusion de nicotine, on s'aperçoit que seules les régions qui contiennent des sites fixant l'ACh consomment un surplus d'énergie [28], ce qui tendrait à prouver que ce sont les récepteurs responsables de l'activité électrique provoquée par la nicotine in vivo. La localisation des divers transcrits, réalisée par hybridation in situ de sondes nucléotidiques (voir par exemple [29]), montre une grande diversité d'expression (figure 3). Chez le rat, tandis qu' $\alpha 4$ et $\beta 2$ sont transcrites dans la quasi-totalité des structures, $\alpha 2$ est restreinte au noyau interpédonculaire, et $\alpha 3$ comme $\beta 4$ se trouvent uniquement dans l'habénula médiale (résultats non publiés) et certains ganglions autonomes.

Scule l'immunohistochimie permet de savoir si une protéine est réellement exprimée et de connaître sa localisation finale (question cruciale quand il s'agit de neurones, possédant des prolongements pouvant atteindre plusieurs centimètres). L'utilisation des anticorps monoclonaux MAb35 et 


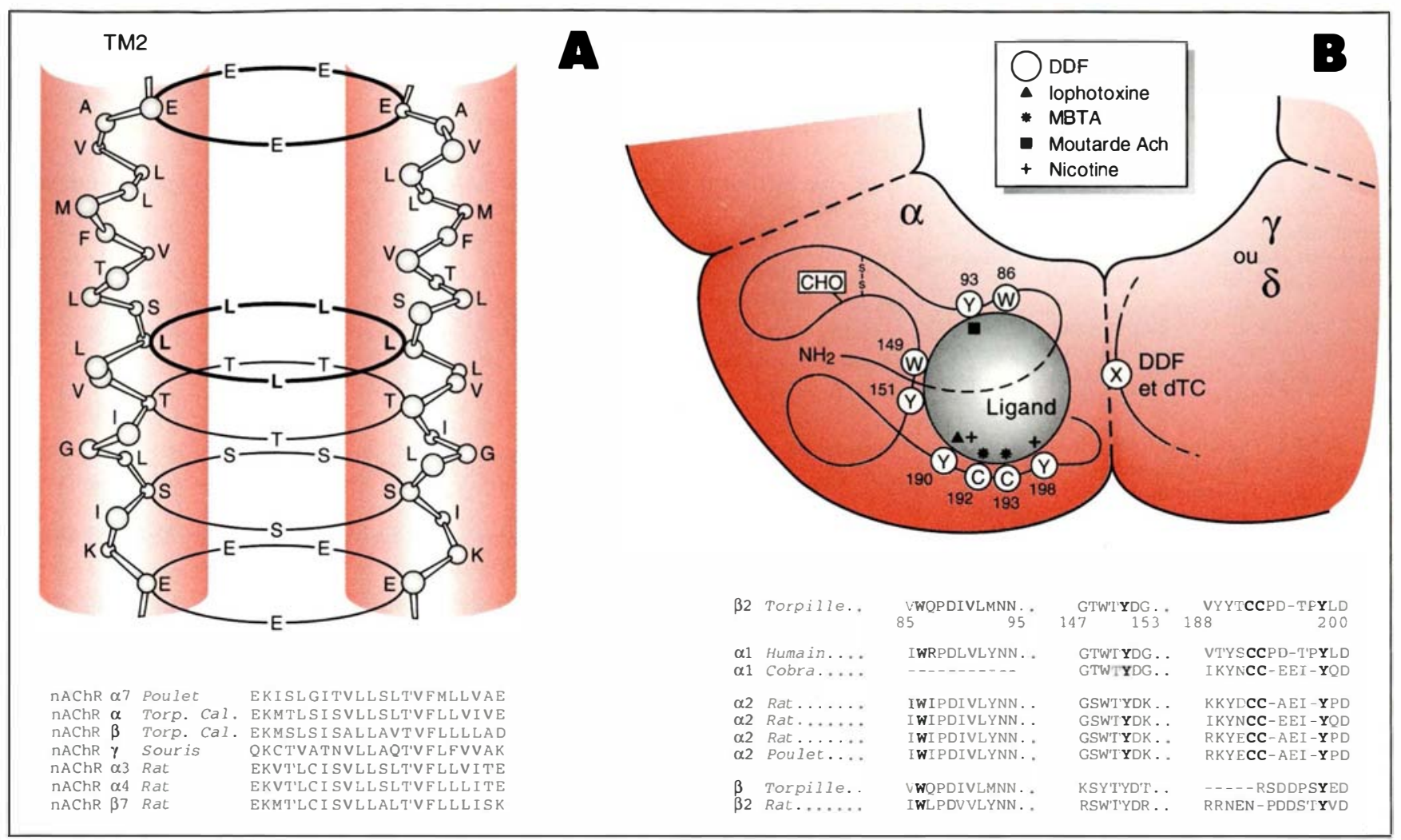

Figure 2. A. Structure présomptive du pore dans le récepteur $\alpha 7$, entouré par le segment MII de chaque sousunité. Séquences alignées constituant le segment MII dans différentes sous-unités nicotiniques. Les acides aminés sont présentés à l'aide de leur code à une lettre. B. Modèle à trois boucles du site de fixation des ligands compétitifs dans le récepteur musculaire. Les acides aminés signalés sur le dessin sont marqués par divers ligands compétitifs. Plusieurs des acides aminés essentiels sont conservés dans différentes sous-unités nicotiniques. dTC: dtubocurarine ; DDF : $p(N, N)$-diméthyl-aminobenzène diazonium ; MBTA : 4-(N-maléimino)-benzyltriméthylammonium ; moutarde ACh : acétate de 2-(N-2'-chloroethyle)-N-methylam-ethyle). (adapté de [5]).

MAb270 montre une répartition des récepteurs à travers tout le cerveau, cohérente avec les études de liaisons (ACh plus $\alpha$-Bgt) [30]. La distribution exacte des sous-unités commence à ĉtre étudiée [31].

$\mathrm{La}$ localisation intracellulaire des récepteurs nicotiniques est également variable.

\section{In vivo le récepteur nicotinique neuronal est fréquemment localisé sur les terminaisons axonales}

Lorsqu'on incorpore de la méthionine ${ }^{35} \mathrm{~S}$ dans la rétine de poisson rouge, on peut isoler des nAChR marqués à partir du tectum optique, ce qui signifie que les récepteurs, synthétisés dans le soma, ont été transportés jusqu'aux boutons synaptiques [32]. Cette localisation présynaptique se $\mathrm{m} / \mathrm{s} n^{\circ} \perp$ vol. 9, janvier 93 rencontre fréquemment dans le système nerveux central. Chez le rat, on en connaît également dans le noyau interpédonculaire, sur les terminaisons provenant de l'habénula, ainsi que dans le striatum où ils semblent moduler la libération de dopamine par les terminaisons venant de la substance noire [33]. Si l'on injecte dans les ventricules de rat de la 6-hydroxydopamine et de la 5,7-dihydroxytryptamine, qui détruisent respectivement les axones dopaminergiques et sérotoninergiques, on observe une réduction marquée des sites nicotiniques dans le striatum (30\%) et l'hypothalamus $(50 \%)$. Cela indique qu'au moins une partie des nAChR situés dans ces structures se trouvent sur des terminaisons à sérotonine ou à dopamine [34]. Dans le cortex préfrontal de rat, les neurones pyramidaux sont innervés par des terminaisons glutamatergi- ques en provenance, par exemple, du thalamus. La nicotine serait susceptible de moduler la libération de glutamate en agissant au niveau des terminaisons axonales de ces fibres (Catherine Vidal, soumis pour publication).

Cependant, diverses données permettent de penser que certains sous-types de récepteurs sont localisés sur les corps cellulaires neuronaux et sur leurs dendrites. Plusieurs structures qui expriment le $n A C h R$ reçoivent effectivement une innervation cholinergique. Par exemple, le cortex cérébral reçoit des projections cholinergiques provenant du noyau basal de Meynert. Or le récepteur $\alpha 4 \beta 2$ semble se trouver dans les neurones pyramidaux qui se projettent vers des cibles sous-corticales. L'ACh pourrait ainsi avoir un rôle facilitateur de la sortie glutamatergique excitatrice du cortex [35]. 


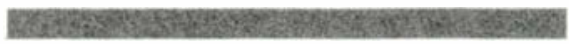
RÉFÉRENCES

24. Schoepfer R, Conroy WG, Whiting P, Gore $\mathrm{M}$, Lindstrom J. Brain $\alpha$-bungarotoxin binding protein cDNAs and MAbs reveal subtypes of this branch of the ligandgated ion channel gene superfamily. Neuron 1990 ; 5 : 35-48.

25. Chiappinelli VA. Kappa-bungarotoxin : a probe for the neuronal nicotinic receptor in the avian ciliary ganglion. Brain Res $1983 ; 277: 9-21$

26. Vidal C, Changeux JP. Pharmacological profile of nicotinic acetylcholine receptors in the rat prefrontal cortex : an electrophysiological study in a slice preparation. Neuroscience 1989 ; 29 : 261-70.

27. Luetje CW, Wada K, Rogers S, Abramson SN, Tsuji K, Heinemann S, Patrick J. Neurotoxins distinguish between different neuronal nicotinic acetylcholine receptor subunit combinations. $J$ Neurochem $1990 ; 55: 632-40$.

28. London ED, Connolly RJ, Szikszay M, Wamsley JK, Dam M. Effects of nicotine on local cerebral glucose utilization in the rat. J Neurosci $1988 ; 8$ : 3920-8.

29. Wada E, Wada K, Boulter J, Deneris E, Heinemann S, Patrick J, Swenson LW. Distribution of alpha2, alpha3, alpha4 and beta2 neuronal nicotinic receptor subunit mRNAs in the central nervous system : a hybridization histochemical study in the rat. J Comp Neurol 1989 ; 284 : 314-35.

30. Swanson LW, Simmons DM, Whiting PJ, Lindstrom J. Immunohistochemical localization of neuronal nicotinic receptors in the rodent central nervous system. $J \mathrm{Neu}$ rosci $1987 ; 7: 3334-42$.

31. Hill JA, Zoli M, Bourgeois JP, Changeux JP. Immunohistochemical localization of a neuronal nicotinic receptor: the $\beta 2$ subunit. J Neurosci 1993 (sous presse).

32. Henley JM, Lindstrom JM, Oswald RE. Acetylcholine receptor synthesis in retina and transport to optic tectum in goldfish. Science $1986 ; 232$ : 1627-9.

33. DiChiara G, Imperato A. Drugs abused by humans preferentially increase synaptic dopamine concentrations in the mesolimbic system of freely moving rats. Proc Natl Acad Sci USA 1988 ; 85 : 5274-78.

34. Schwartz RD, Lehmann J, Kellar KJ. Presynaptic nicotinic cholinergic receptors labeled by $\left[{ }^{3} \mathrm{H}\right]$ acetylcholine on catecholamine and serotonin axons in brain. $J \mathrm{Neu}$ rochem $1984 ; 42$ : 1495-8.

35. Bravo $\mathrm{H}$, Karten HJ. Pyramidal neurons of the rat cerebral cortex, immunoreactive to nicotinic acetylcholine receptors, project mainly to subcortical target. J Comp Neurol 1992 ; 320 : 62-8.

Noyau basal de Meynert : principal groupe de neurones cholinergiques envoyant des terminaisons dans le cortex cérébral.

Potentialisation : augmentation d'activité due à un facteur extérieur au système considéré.

Voie habénulo-interpédonculaire : circuit limbique d'intérêt particulier pour l'étude de la transmission cholinergique centrale; en effet, l'habénula reşoit des afférences cholinergiques du septum et en envoie vers l'interpédonculaire. De plus, toutes les sous-unités neuronales du récepteur nicotinique se trouvent soit dans l'habénula, soit dans le noyau inter pédonculaire.

Voie nigro-striée : un des principaux composants des circuits extrapyramidaux impliqués dans le contrôle de la motricité. Elle relie la substance noire et le caudé-putamen.

Voie mésocorticolimbique : voie reliant l'aire tegmentale ventrale principalement au noyau accumbens et au tubercule olfactif.

Dépendant du voltage : se dit d'une caractéristique biochimique ou électrophysiologique qui varie en fonction du potentiel transmembranaire.

La localisation fréquemment axonale des récepteurs rend difficile l'étude de leur activité car l'analyse électrophysiologique sur les terminaisons est une technique en cours de développement. Des études électrophysiologiques récentes ont cependant permis de caractériser les récepteurs neuronaux et de les comparer au nAChR musculaire.

\section{Le calcium est un ion perméant et un régulateur allostérique du récepteur nicotinique neuronal}

Comme le récepteur musculaire, les nAChR neuronaux contiennent des canaux cationiques non spécifiques [36], délivrant un courant entrant rapide par bouffées de 5 à $10 \mathrm{~ms}$. Toutefois, ils possèdent des propriétés particulières qui pourraient se révéler essentielles pour leur fonction. A la différence du récepteur musculaire, la probabilité d'ouverture du canal dépend du potentiel électrique transmembranaire [37]. Le potentiel d'action parcourant un axone, et d'une manière générale le potentiel de membrane, peut donc modifier l'efficacité de réponse des nAChR. En retour, la dépolarisation provoquée par les nAChR peut affecter la propagation des potentiels d'action en inactivant les canaux dépendants du voltage perméables au sodium et au calcium.

Une particularité importante du nAChR neuronal est sa perméabilité au calcium. Celle-ci est plus importante que celle du nAChR musculaire et très proche de celle du récepteur NMDA au glutamate $[36,38]$. Ce flux calcique peut être amplifié par une cascade dépendante du voltage (via des canaux $\mathrm{Ca}^{2+}$ ouverts par la dépolarisation due à l'activation des nAChR puis par un effet calciuminduced-calcium-released) [39]. Les conséquences de cette propriété sont nombreuses. D'une part, l'action finale du nAChR pourrait parfois être inhibitrice par activation de conductances potassium $[40,41]$ et chlore [36] dépendantes du calcium. D'autre part, les ions calcium entrés par le canal nicotinique pourraient jouer un rôle de second messager, augmentant l'exocytose d'autres transmetteurs (si le récepteur se trouve sur le bouton synaptique), ou activant des facteurs de transcription sensibles au calcium [42] (si le récepteur se trouve sur le corps cellulaire).

Le calcium extracellulaire modifie également les propriétés du récepteur selon deux modalités : il diminue la conductance élémentaire aux potentiels négatifs, ce qui pourrait être dû à un site de fixation dans le canal (ou dans le vestibule de celui-ci), et il potentialise l'activité du récepteur en 


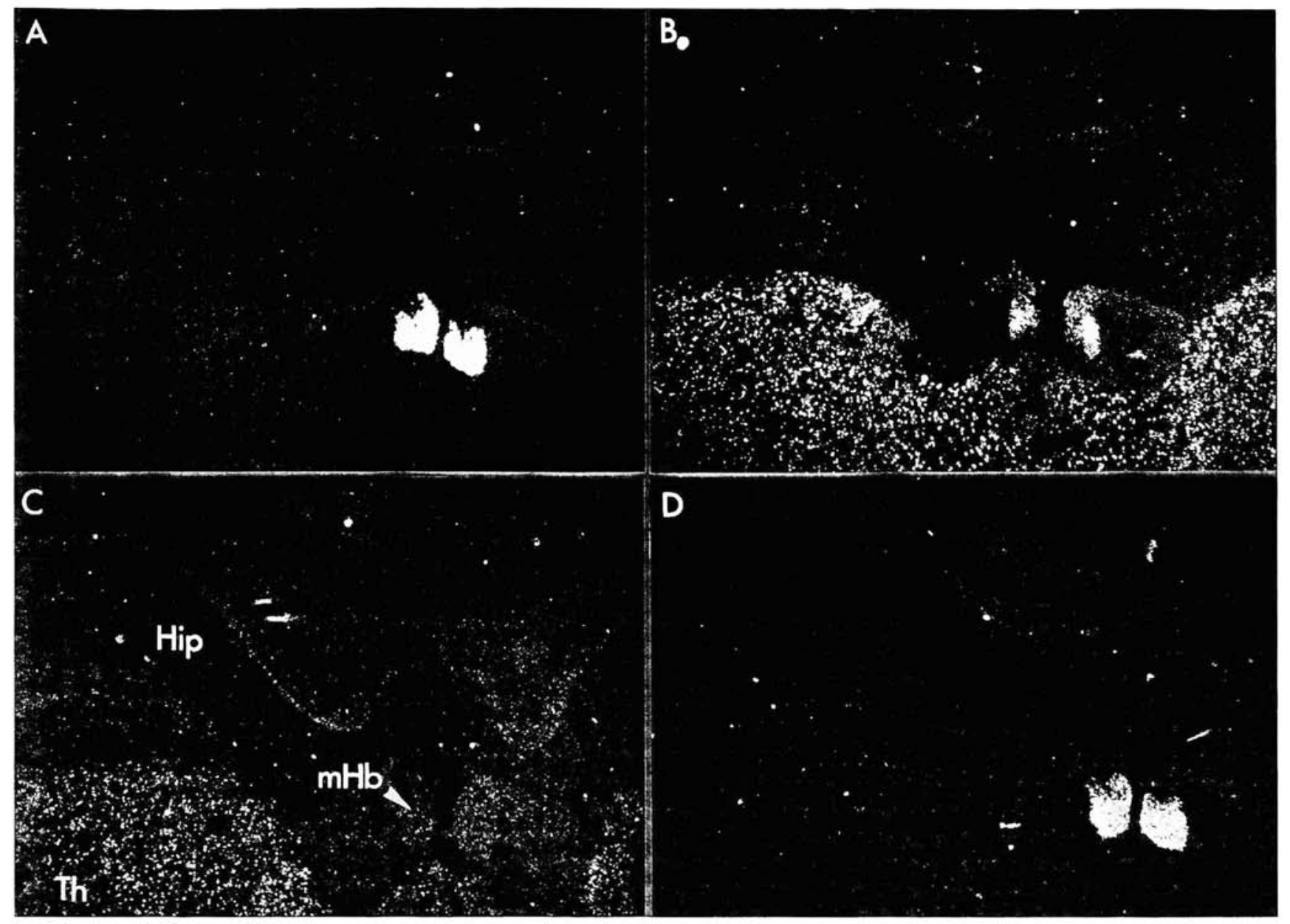

Figure 3. Distribution de divers transcrits de nAChR visualisés par hybridation in situ de sondes oligodésoxynucléotidiques sur le cerveau de rat. A- $\alpha 3$. B- $\alpha 4$. C- $\beta 2$. D- $\beta 4$. Hip : hippocampe; mHb : habénula médiale; Th: thalamus.

augmentant sa probabilité d'ouverture, propriété qui semble liée à la présence d'un site allostérique [43].

\section{Régulation des propriétés du récepteur nicotinique par I'AMPc et de son abondance par l'innervation}

Dans des neurones de ganglion cilié de poulet, l'AMPc augmente la réponse nicotinique sans toutefois changer le nombre de récepteurs détectables à l'aide d'anticorps. La constante d'affinité pour l'ACh ainsi que la conductance élémentaire ne sont pas modifiées. Il semble donc que seul le rapport entre états conformationnels allostériques du récepteur (fonctionnels/non fonctionnels) soit affecté par le nucléotide [44].

$\mathrm{m} / \mathrm{s} n^{\circ} 1$ vol. 9, janvier 93
Divers facteurs physiologiques modifient l'abondance du nAChR neuronal ainsi que le type exprimé. Chez le poulet, l'apparition de récepteur dans certaines structures coïncide avec l'arrivée des fibres cholinergiques afférentes, ce qui pourrait signifier que l'innervation est nécessaire à l'expression des gènes codant pour les sous-unités du récepteur [45]. L'innervation règle également le type de récepteur synthétisé. Dans les ganglions sympathiques de grenouille, les cellules de types B et C sont innervées par des fibres qui peuvent être distinguées et possèdent des constantes d'inactivation du courant induit par l'ACh différentes. Si l'on provoque l'innervation de cellules $B$ par des fibres $C$, l'inactivation du courant des cellules B devient de type $C$ [46]. L'existence de ces diverses régulations suggère l'importance physiologique du nAChR neuronal. Cette importance est soulignée par les modifications d'activité dont il est l'objet au cours du développement de certaines affections.

\section{Récepteur nicotinique et pathologie}

Bien que le nAChR puisse être affecté lors de plusieurs affections neurologiques (par exemple le syndrome d'Alzheimer (références dans [47])), la principale affection associée est bien entendu le tabagisme. Le tabac est un des principaux facteurs de morts accidentelles en France, à la suite de maladies cardiovasculaires et de tumeurs de l'appareil bucco-respiratoire (c'est la première cause de cancer : $24 \%$ 


\section{RÉFÉRENCES}

36. Mulle C, Choquet D, Korn H, Changeux JP. Calcium influx through nicotinic receptor in rat central neurons : its relevance to cellular regulation. Neuron $1992 ; 8$ : 135-43.

37. Sands SB, Barish ME. Neuronal nicotinic acetylcholine receptor currents in pheochromocytoma (PC12) cells : dua mechanisms of rectification. J Physiol 1992 ; $447: 467-87$.

38. Vernino S, Amador M, Luetje CW, Patrick J, Dani JA. Calcium modulation and high calcium permeability of neuronal nicotinic acetylcholine receptors. Neuron $1992 ; 8: 127-34$.

39. Vijayaraghavan S, Pugh PC, Zhang Z, Rathouz MM, Berg DK. Nicotinic receptors that bind $\alpha$-bungarotoxin on neurons raise intracellular free $\mathrm{Ca}^{2+}$. Neuron 1992 ; $8: 353-62$

40. Wong LA, Gallagher JP. Pharmacology of nicotinic receptor-mediated inhibition in rat dorsolateral septal neurons. J Physiol $1991 ; 436: 325-46$.

41. Fuchs PA, Murrow BW. Cholinergic inhibition of short (outer) hair cells of the Chick's cochlea. J Neurosci $1992 ; 12$ : 800-9.

42. Sheng $M$, McFadden G, Greenberg ME. Membrane depolarization and calcium induce c-fos transcription via phosphorylation of factor CREB. Neuron 1990; 4 : 571-82.

43. Mulle C, Lena C, Changeux JP. Potentiation of nicotinic acetylcholine receptor response by external calcium in rat central neurons. Neuron $1992 ; 8$ : 937-45.

44. Margiotta JF, Berg DK, Dionne VE. Cyclic AMP regulates the proportion of functional acetylcholine receptors on chicken ciliary ganglion neurons. Proc Natl Acad Sci USA $1987 ; 84: 8155-59$

45. Daubas P, Devillers-Thićry A, Geoffroy B, Martinez S, Bessis A, Changeux JP Differential expression of the neuronal acetylcholine receptor $\alpha 2$ subunit gene during chick brain development. Neuron 1990 ; 5 : 49-60

46. Marshall LM. Presynaptic control of synaptic channel kinetics in sympathetic neurons. Nature $1985 ; 317$ : 621-3.

47. Sunderland T, Tariot PN, Newhouse $P$. Differential responsivity of mood, behavior and cognition ot cholinergic agents in elderly neuropsychiatric populations. Brain Res Rev $1988 ; 13: 371-89$.

48. Cachin Y, Dreyfus JC. Statistiques de mortalitć en France pour 1990. Les cancers comparćs aux autres causes. médecine/sciences $1992 ; 8: 863-65$.

49. Ochoa ELM, LI L, McNamee MG Desensitization of central cholinergic mechanisms and neuroadaptation to nicotine. Mol Neurobiol $1990 ; 4: 251-87$.
[48]). Comme toutes les drogues à accoutumance, la nicotine entraîne une tolérance et une dépendance tant comportementale (addiction) que physique (dependence). La neuroadaptation à la nicotine a fait l'objet de nombreux travaux (revue dans [49]). La dépendance physique, responsable des symptômes du manque lors du sevrage, est probablement en relation avec le système dopaminergique mésocorticolimbique que l'on estime être un des vecteurs de la satisfaction naturelle (sexe ou nutrition) ou artificielle (opiö̈des, alcool, etc.) [50]. La prise de nicotine affecte les neurotransmetteurs du système comme leurs récepteurs. A l'instar d'autres drogues à accoutumance, la nicotine augmente transitoirement la quantité de dopamine extracellulaire dans le noyau accumbens. Cet effet est bloqué par la mécamylamine, antagoniste des nAChR cérébraux [33]. De plus, l'administration chronique de nicotine augmente la synthèse de dopamine de manière persistante (augmentation encore significative après plusieurs semaines de manque) dans le noyau accumbens et l'hippocampe, effet également supprimé par la mécamylamine [51]. L'administration chronique de nicotine modifie également l'activité des récepteurs du système. Dans le striatum, par exemple, elle entraîne la désensibilisation des récepteurs dopaminergiques présynaptiques D2 (situés sur les terminaisons en provenance de la substance noire) [52] et une augmentation du nombre de sites nicotiniques. On n'observe cependant pas d'augmentation significative du taux de transcription de $\alpha 2,3,4,5$ et de $\beta 2$, ce qui implique le recrutement d'une réserve de récepteurs antérieurement intracellulaires ou inactifs [53].

Une meilleure compréhension de la physiologie du nAChR permettrait sans doute d'appréhender plus concrètement la dépendance à la nicotine et éventuellement de la traiter.

\section{Conclusion}

Les récepteurs nicotiniques de l'acétylcholine forment une famille de molécules polymériques. Les différentes sous-unités neuronales présentent une remarquable conservation de séquence et de structure entre elles et avec celles du récepteur musculaire. L'homogénéité de la famille est renforcée par le fait que $\beta 2$ ou $\beta 4$ peuvent remplacer $\beta 1$ et, avec $\alpha 1$, $\gamma$ et $\delta$, donner des récepteurs fonctionnels. Les études pharmacologiques du récepteur, tant in vitro qu'in vivo, montrent une diversité de comportements qui s'avèrent en relation avec l'identité des sous-unités le composant. L'électrophysiologie confirme ce fait puisque la cinétique du courant et la conductance du canal dépendent des sous-unités qui le forment. Le neurone dispose ainsi d'une gamme d'ingrédients avec lesquels il peut composer les mélanges lui convenant le mieux. La situation anatomique des sous-unités met cependant en relief des inhomogénéités dans la fréquence des récepteurs utilisés puisque plus de $90 \%$ appartiennent à un seul type. La régulation spatio-temporelle de leur manifestation signale l'importance physiologique des diverses formes de nAChR.

De par son comportement vis-à-vis du calcium et son implication dans des troubles neurologiques, le récepteur nicotinique neuronal de l'acétylcholine reste un modèle pour la recherche fondamentale en électrophysiologie des canaux ioniques comme pour la recherche thérapeutique en psychopharmacologie

\section{Remerciements}

Les auteurs remercient Jean-Pierre Changeux, Anne Devillers-Thiéry, Jean-Luc Galzi et Fabienne Guggiari pour leur collaboration.

\section{TIRÉS A PART}

N. L.e Novère. 


\section{RÉFÉRENCES}

50. Koob GF, Bloom FE. Cellular and molecular mechanisms of drug dependence. Science 1988 ; 242 : 715-23.

51. Mitchell SN, Brazcll MP, Joscph MH, Alavijch MS, Gray JA. Regionally specific effects of acute and chronic nicotinc on rates of catecholamine and 5-hydroxytryptamine synthesis in rat brain. Eur J Pharmacol 1989 ; $167: 311-22$

52. Harsing LG Jr, Sershen H, Lajtha A. Dopaminc cfflux from striatum after chronic nicotine : evidence for autoreceptor descnsitization. J Neurochem 1992; 59 : 48-54.

53. Marks MJ, Pauly JR, Gross SI, Dencris ES, Hermans-Borgmeycr I, Neincmann SF, Collins AC. Nicotine binding and nicotinic receptor subunit RNA after chronic nicotinc treatment. J Neurosci $1992 ; 12$ : 2765-84.

54. Conroy G, Vernallis AB, Berg DK. The $\alpha 5$ Gene product assembles with multiple acctycholine receptor subunits to form distinctive receptor subtypes in brain. Neuron 1992 ; 9 : 679-91.

55. Galzi M, Revah F, Bcssis A, Changcux JP. Functional architecture of the nicotinic acctyl choline receptor : from elcctric organ to brain. Annu Rev Pharmacol 1991; 31 37-72.

\section{Summary}

Neuronal nicotinic acetylcholine receptor : from gene to smoking addiction

The nicotinic acetylcholine receptor present in muscle is an allosteric pentameric protein and has been used as a model for the structure and function of the superfamily of ligand gated ion channels. A related family of receptors is localized in the nervous system. These receptors, which are composed of two $\alpha$ subunits that are chiefly responsible for the binding of acetylcholine and three non- $\alpha$ subunits, mediate neuronal nicotinic neurotransmission. To date, ten genes have been isolated encoding subunits of the neuronal nicotinic receptor expressed in various brain regions. The electrophysiological and pharmacological properties of the neuronal nicotinic receptors are dependent upon the subunit composition of the receptor, and can be modified by innervation as well as by agents such as calcium and cAMP. The expression of the receptor can be altered as a result of diverse neuronal pathologies.

\section{FLASH}

\section{JEAN-PIERRE CHANGEUX, PRIX LOUIS JEANTET 1993}

Jean-Pierre Changeux, professeur au Collège de France et directeur du laboratoire de Neurobiologie Moléculaire à I'Institut Pasteur de Paris, est I'un des trois lauréats du Prix Louis Jeantet 1993.

II est récompensé pour sa contribution éminente à l'élucidation de la structure et de la fonction du récepteur canal nicotinique de l'acétylcholine. Rappelons que cette consécration vient après la remise à Jean-Pierre Changeux de la médaille d'or du CNRS, en 1992. 